\title{
Index of Inequality-Adjusted Happiness (IAH) Improved: A Research Note
}

\author{
Wim Kalmijn • Ruut Veenhoven
}

Published online: 19 September 2013

(C) Springer Science+Business Media Dordrecht 2013

\begin{abstract}
Eight years ago we proposed a new measure of happiness in nations, called Inequality-Adjusted Happiness (IAH). This measure indicates how successful nations are in combining a high level of happiness and a low degree of inequality in happiness among citizens. The index gives equal weight to the level of happiness measured using the mean and inequality of happiness measured using the standard deviation. Scores on this index are now available for 139 nations. In this paper we present a technical improvement for the method to calculate IAH.
\end{abstract}

Keywords Happiness · Inequality · Inequality-Adjusted Happiness (IAH)

\section{Dual Policy Aims}

Happiness is a rising topic on the political agenda and this brings back a classic question on priorities in the promotion of happiness. Should we focus on achieving a higher level of happiness, or reduce inequality in happiness in the first place?

Progress in creating greater happiness is typically measured using survey studies, in which a representative sample of the general public is asked to answer a question such as 'Taking all together, how satisfied are you with your life as-a-whole these days? Please respond by choosing a number between 0 and $10^{\prime} .1$

The level of happiness is then expressed by the mean reported happiness, e.g. in studies by Diener (2000), Diener et al. (2010), Tay and Diener (2011). Comparison of the findings

\footnotetext{
${ }^{1}$ Questions on happiness can be phrased in more ways, using different key words. An overview of questions that fit the concept of 'the subjective appreciation of one's life as-a-whole is available in Veenhoven (2011b). The method described in this paper applies to all measures.
}

W. Kalmijn · R. Veenhoven $(\bowtie)$

Erasmus University Rotterdam, Rotterdam, The Netherlands

e-mail: veenhoven@ese.eur.nl 
over time shows that average happiness has increased over the last decennium in most countries (Veenhoven and Hagerty 2006).

Likewise progress in reducing inequality can be measured using the standard deviation ${ }^{2}$ of responses to survey questions about happiness. Research shows that disparity in happiness has decreased substantially during the last decennium in modern nations (Veenhoven 2005).

\section{Need for Inclusive Monitoring}

Achievement of one of these aims may come at the expense of the other, for instance when greater happiness of the majority is brought about by exploitation of a minority, or when greater equality results in unhappiness for the majority, as has happened in former communist countries. Though current social policies appear to add both to a higher level of happiness and to smaller inequality in happiness (Ott 2005), it is worth keeping an eye on the combination. This is common practice in policy making where typically multiple goals are involved. In this case it calls for an indicator that combines both the aim of a higher level of happiness and that of a more equitable distribution of happiness.

\section{Inequality-Adjusted Happiness}

A first attempt to combine the estimated average happiness $(m)$ and the within nation standard deviation ( $s$ ) was made by Veenhoven (2003), who chose the simple ratio $\mathrm{m} / \mathrm{s}$. This statistic, however, has several disadvantages, the most important being that it does not allow us to control the relative weights assigned to the utilitarian and egalitarian views on happiness, focusing exclusively on the level $(m)$ and the dispersion (s) respectively.

In an earlier paper in this journal (Veenhoven and Kalmijn 2005), we presented a solution of this problem. To this end, we introduced an indicator which we called Inequality-Adjusted Happiness $(I A H)$ which is defined as an integer number ranging from 0 to 100 . This index is usually applied to the specific case of equal weights assigned to both views on happiness.

Each nation can be represented in Fig. 1 as a point with abscissa $m$ and ordinate $s$. The average value $m$ is bounded between the two terminal values of the scale of measurement, represented by the points $\mathrm{L}$ and $\mathrm{H}$ respectively. It can be proven (Kalmijn 2010) that for each value of $m$ the standard deviation $s$ has a maximum possible value and that only points within or on the circumference of the semicircle with diameter LH can represent the happiness situation in a nation.

The $I A H$-value 100 corresponds to the best possible combination, which is characterized by the highest possible scale value of $m$ and $s=0$; the point $\mathrm{H}$ in Fig. 1. The situation $I A H=0$ is a compromise between both views of the worst conceivable situation, which is $\mathrm{L}$ according to the utilitarians and $\mathrm{T}$ in the extremely egalitarian view. This compromise is represented by a point $\mathrm{W}$ on the semicircle circumference somewhere between $\mathrm{L}$ and $\mathrm{T}$. Its

\footnotetext{
${ }^{2}$ In an earlier paper in this journal we argued that the standard deviation is a proper statistic for measuring inequality in happiness (Kalmijn and Veenhoven 2005). Recently, Delhey and Kohler (2011) proposed an adjustment to that method. We advised against that adjustment in Kalmijn (2012) and Veenhoven (2012) to which Delhey and Kohler (2012) replied.
} 


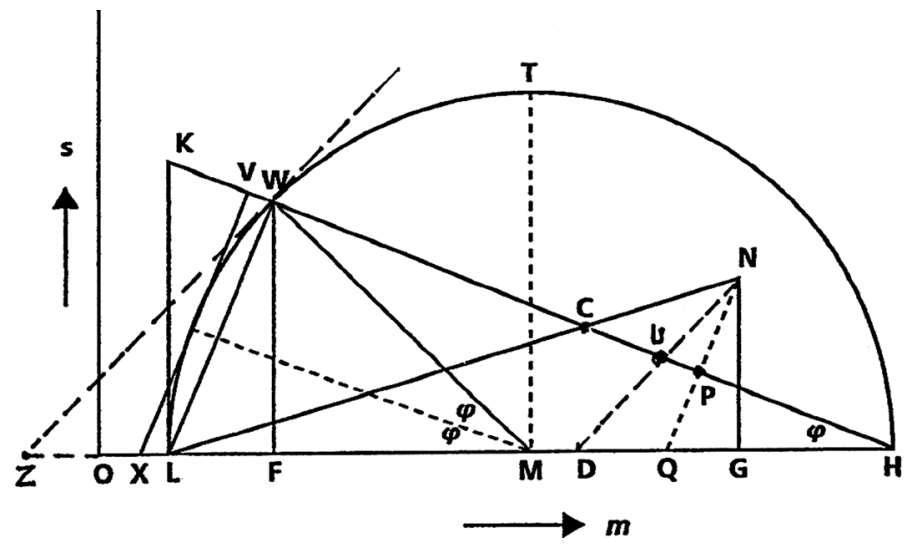

Fig. 1 Three projections of $N$ on the $I A H$-axis $W H$ : central $(C)$, orthogonal $(P)$ and skew $(U)$

exact position depends on the weights assigned to both views: the more weight is given to the utilitarian view, the closer $\mathrm{W}$ is to $\mathrm{L}$.

The $I A H$-value of a nation represented by a point $\mathrm{N}$ with $m$ and $s$ as its Cartesian coordinates, is obtained by projecting the point $\mathrm{N}$ onto the line segment $\mathrm{WH}$, which acts as the 'IAH axis'. The exact position of the projection depends on the type of projection.

Two options were considered in the 2005 paper, central projection and orthogonal projection, resulting in the projections $\mathrm{C}$ and $\mathrm{P}$ respectively of $\mathrm{N}$. The final choice was made in favor of the orthogonal projection and in this case $I A H$ is a linear combination of $m$ and $s$.

\subsection{Data on IAH in Nations}

Using this approach $I A H$ scores have been computed for most nations of the world. These data are presented in the World Database of Happiness (Veenhoven 2011a) in two reports that are continuously updated. In the first report a rank list of current version of IAH in 139 nations is presented (Veenhoven 2011c). On this list Costa Rica is currently at the top of the rank with an $I A H$ score of 79 and Tanzania at the bottom with a score of 19 . In the second report findings on changes in $I A H$ since 1973 in 14 western nations are presented, with a rise in 10 nations and a decline in four (Veenhoven 2011d).

This is how things were up to 2011. The definition of IAH and how this statistic is computed was changed slightly in 2012. In this paper we explain why this modification was made and discuss the differences in results.

\section{The Problem}

A problem arises with nations that might be represented by a point within the semicircle segment LW. Since LW and WH are orthogonal, any nation represented by a point on the line segment $\mathrm{LW}$ is projected orthogonally onto the $I A H$-axis in the point $\mathrm{W}$. As the reader can verify easily, a nation represented by a point to the left of the line segment LW, but within the semicircle, will be projected onto the $I A H$-axis as a point between $\mathrm{V}$ and $\mathrm{W}$, so outside the semicircle. 
The $I A H$-value of these nations is less than that of the compromise situation W. So to avoid negative $I A H$-values, we decided to assign the value $I A H=0$ to a nation projected onto $\mathrm{V}$ and to accept that the $I A H$-value of $\mathrm{W}$ was a small positive number (about +4$)$. In this way-out, the IAH-value of the nation $N$ is defined as $100 \times \underline{V P} / \underline{V H}$, and rounded to integer values.

Although the practical consequences of the above decisions seem to be rather modest, at least in the 'equal weights case', the solution described above was felt to be unsatisfactory, but acceptable as long as no better alternative was available.

\section{The Solution: Skew Projection}

In a later discussion on this statistic, Dr. Jan Koster suggested using a skew projection as an alternative that enables one to circumvent the above problem. In the suggested method, the projector of $\mathrm{N}$ is drawn parallel to the tangent $\mathrm{WZ}$ in $\mathrm{W}$ to the semicircle. The point of intersection $\mathrm{U}$ of the line segment $\mathrm{WH}$ and this projector through $\mathrm{N}$ defines the 'improved' $I A H$-value of the nation $N$ as $100 \times \underline{W U} / W H$, and rounded to integer values. In this way, projection outside the semicircle is impossible.

\subsection{Consequences of the Application of the Skew Projection Method}

The most important consequence is that the point $\mathrm{W}$ has the zero $I A H$-value it should have and all other $I A H$-values are positive.

\subsection{Formula}

The improved $I A H$-values can be calculated using a formula that is derived in Appendix 1. In the case equal weights are assigned to both the utilitarian and the egalitarian view, and happiness is quantified on a $0-10$ range, the $I A H$-value can be simply approximated as:

$$
I A H \approx 8.28 \cdot(m-s)+17.2 .
$$

\subsection{Example}

Consider the case of China, where mean happiness on scale $0-10$ is 6.14 and the standard deviation 2.45 (cf. Appendix 2). Substitution of these values gives:

$$
I A H \approx 8.28 \cdot(6.14-2.45)+17.2=47.8, \text { which is rounded to } 48 .
$$

\subsection{Application to Available Data}

The modified $I A H$-values of a selection of 15 out of 139 nations are listed in Appendix 2 as an illustration together with the corresponding original values within parentheses. We selected the five nations with the highest and the five with the lowest modified $I A H$-values and added five more nations with intermediate values. The mean values and the corresponding standard deviations of these nations are average values of survey results for the period 2003-2009. Nations with one single survey only in this period were ignored in the selection of data for Appendix 2. 
The numerical $I A H$-values of all the 15 nations are slightly smaller than the original ones, due to the shift of the point with $I A H=0$ towards the point $\mathrm{H}$. For most nations the magnitude of the difference between the two rounded numbers is of the order of 4 units, as might be expected. The values of the new statistic give a more valid indication of the extent to which the happiness situation in a nation is remote from the 'ideal' situation represented by the point $\mathrm{H}$. Therefore, we have decided to adopt this new statistic in the World Database of Happiness.

The most recent $I A H$ values are available in the World Database of Happiness, in the finding reports on IAH in Nations (Veenhoven 2013a, b).

\section{Conclusion}

The application of a skew projection results in IAH values with properties superior to those of the originally defined index and we recommend that these are replaced with an IAHvalue calculated on the basis of the skew projection method as described in this paper.

Acknowledgments The authors wish to thank Dr. Jan Koster (Erasmus University Rotterdam) for his suggestion to apply the skew projection method.

\section{Appendix 1: Improved $I A H$ by the Application of Skew Projection}

Let in Fig. 1 the point $\mathrm{M}$ be the centre of the semicircle with radius $L M=r$. If $\mathrm{L}$ is adopted as the origin, a nation $\mathrm{N}$ can be represented by its mean happiness value $m=L G$ as its abscissa and its internal standard deviation $s=\underline{N G}$ as its ordinate.

Let $\mathrm{W}$ represent the compromise for the worst conceivable situation and $W Z$ be the tangent through $W$ to the semicircle. The skew projection $\mathrm{U}$ of $\mathrm{N}$ onto the $I A H$-axis $\mathrm{WH}$ is obtained as the intersection of WH with the line segment ND through N parallel to WZ.

In that case, the $I A H$-value of $\mathrm{N}$ equals the ratio $(U W / H W) \times 100$.

From the parallelism of $\mathrm{WZ}$ and UD follows the proportionality

$$
\underline{U W} / \underline{H W}=\underline{Z D} / \underline{Z H} .
$$

The angles $Z M W$ and $D N G$ are equal; let their value be $2 \varphi$, where $\varphi:=\operatorname{angle}(W H L)$. The value of $2 \varphi$ equals $\left[w_{\mathrm{E}} /\left(w_{\mathrm{E}}+w_{\mathrm{U}}\right)\right](\pi / 2)$, where $w_{\mathrm{E}}$ and $w_{\mathrm{U}}$ are the weights assigned to the egalitarian and utilitarian views respectively. Since

$$
\begin{aligned}
\underline{Z D} & =\underline{Z G}-\underline{D G}=\underline{Z L}+\underline{L G}-\underline{D G}=\underline{Z M}-\underline{M L}+\underline{L G}-\underline{D G} \\
& =r / \cos 2 \varphi-r+m-s \tan 2 \varphi \text { and } \\
\underline{Z H} & =\underline{Z M}+\underline{M H}=r / \cos 2 \varphi+r,
\end{aligned}
$$

the $I A H$-value of the nation represented by the point $N(m, s)$ equals

$$
\frac{\frac{r}{\cos 2 \varphi}-r+m-s \tan 2 \varphi}{\frac{r}{\cos 2 \varphi}+r} \times 100
$$

This result can also be written as

$$
I A H=\frac{(m-r) \cos 2 \varphi+r-s \sin 2 \varphi}{r+r \cos 2 \varphi} \times 100
$$


In the case of equal weights $w_{\mathrm{E}}=w_{\mathrm{U}}$ and $2 \varphi=\pi / 4$; when happiness is quantified on a $[0,10]$ scale, then $r=5$ and in this particular case this formula can be simplified to $I A H \approx 8.28(m-s)+17.2$.

\section{Appendix 2: Inequality-Adjusted Happiness (IAH) in 15 Nations 2003-2009}

IAH-values in modified and previous version.

\begin{tabular}{|c|c|c|c|c|}
\hline \multirow[t]{2}{*}{ Nation } & \multicolumn{2}{|c|}{ Happiness } & \multicolumn{2}{|c|}{ Inequality-Adjusted Happiness } \\
\hline & Average & SD & IAH (new) & IAH (old) \\
\hline Denmark & 8.03 & 1.53 & 71 & $(75)$ \\
\hline Iceland & 7.87 & 1.66 & 69 & $(73)$ \\
\hline Switzerland & 7.74 & 1.58 & 68 & $(72)$ \\
\hline Finland & 7.61 & 1.56 & 67 & $(71)$ \\
\hline Netherlands & 7.33 & 1.37 & 67 & $(69)$ \\
\hline Japan & 6.35 & 1.91 & 54 & $(57)$ \\
\hline France & 6.45 & 2.11 & 53 & $(58)$ \\
\hline Indonesia & 6.16 & 2.05 & 51 & $(55)$ \\
\hline Poland & 6.26 & 2.29 & 50 & $(55)$ \\
\hline China & 6.14 & 2.45 & 48 & $(53)$ \\
\hline Macedonia & 4.68 & 2.57 & 35 & (39) \\
\hline Bulgaria & 4.46 & 2.41 & 34 & (37) \\
\hline Mali & 4.73 & 2.77 & 33 & (38) \\
\hline Zimbabwe & 3.23 & 2.28 & 25 & (26) \\
\hline Tanzania & 3.03 & 2.76 & 19 & (22) \\
\hline
\end{tabular}

Source: Veenhoven (2011a, 2013a)

\section{References}

Delhey, J., \& Kohler, U. (2011). Is happiness inequality immune to income inequality? New evidence through instrument-effect-corrected standard deviations. Social Science Research, 40, 742-756.

Delhey, J., \& Kohler, U. (2012). Happiness inequality. Adding meaning to numbers-A reply to Veenhoven and Kalmijn. Social Science Research, 41, 731-734.

Diener, E. (2000). Subjective well-being: The science of happiness and a proposal for a national index. American Psychologist, 55(1), 34-43.

Diener, E., Ng, W., Harter, J., \& Arora, R. (2010). Wealth and happiness across the world: Material prosperity predicts life evaluation, whereas psychosocial prosperity predicts positive feeling. Journal of Personality and Social Psychology, 99, 52-61.

Kalmijn, W. M. (2010). Quantification of happiness inequality, $\mathrm{PhD}$ dissertation Erasmus University Rotterdam (NL) (pp. 182-183). Available at http://repub.eur.nl/resource/pub_21777/index.html.

Kalmijn, W. M. (2012). Happiness is not normally distributed: A comment to Delhey and Kohler. Social Science Research, 41, 100-202.

Kalmijn, W. M., \& Veenhoven, R. (2005). Measuring inequality of happiness in nations: In search for proper statistics. Journal of Happiness Studies, 6, 357-396.

Ott, J. (2005). Level and inequality of happiness in nations: Does greater happiness of a greater number imply greater inequality in happiness? Journal of Happiness Studies, 6, 397-420. 
Tay, L., \& Diener, E. (2011). Needs and subjective well-being around the world. Journal of Personality and Social Psychology, 101, 354-365.

Veenhoven, R. (2003). Equality-adjusted happiness in nations. Paper presented at the conference of the International Society for Quality of Life Studies (ISQOLS), Frankfurt (DE).

Veenhoven, R. (2005). Return of inequality in modern society? Test by dispersion of life satisfaction across time and nations. Journal of Happiness Studies, 6, 457-487.

Veenhoven, R. (2011a). World Database of Happiness: Archive of research findings on subjective enjoyment of life. Erasmus University Rotterdam (NL). Available at: http://worlddatabaseofhappiness.eur.nl.

Veenhoven, R. (2011b). Measures of happiness. World Database of Happiness. Available at: http:// worlddatabaseofhappiness.eur.nl/hap_quer/hqi_fp.htm.

Veenhoven, R. (2011c). Inequality-Adjusted Happiness in 139 nations 2000-2009. World Database of Happiness. Accessed on 15 Aug 2011 at: http://worlddatabaseofhappiness.eur.nl/hap_nat/findingreports/ RankReport_InequalityHappiness.php.

Veenhoven, R. (2011d). Trends Inequality-Adjusted Happiness in nations 1946-2010. World Database of Happiness. Accessed on 15 Aug 2011 at: http://worlddatabaseofhappiness.eur.nl/hap_nat/findingreports/ TrendReport_InequalityAdjustedHappiness.pdf.

Veenhoven, R. (2012). The medicine is worse than the disease: Comment on Delhey and Kohler's proposal to measure inequality in happiness using 'Instrument-Effect-Corrected' standard deviations. Social Science Research, 41, 203-204.

Veenhoven, R. (2013a). Inequality-Adjusted Happiness in 139 nations 2000-2009. World Database of Happiness. Accessed 15 May 2013 at, http://worlddatabaseofhappiness.eur.nl/hap_nat/findingreports/ RankReport_InequalityHappiness.php.

Veenhoven, R. (2013b). Trends Inequality-Adjusted Happiness in nations 1946-2010, World Database of Happiness. Accessed 15 May 2013 at, http://worlddatabaseofhappiness.eur.nl/hap_nat/findingreports/ TrendReport_InequalityAdjustedHappiness.pdf.

Veenhoven, R., \& Hagerty, M. (2006). Rising happiness in nations 1946-2004. A reply to Easterlin. Social Indicators Research, 79, 421-436.

Veenhoven, R., \& Kalmijn, W. M. (2005). Inequality-Adjusted Happiness in nations: Egalitarianism and utilitarianism married in a new index of societal performance. Journal of Happiness Studies, 6, 421-455. 